\title{
Effect of age and body condition score on sperm production potential among some indegenous bull cattle in Mubi Adamawa State, Nigeria
}

\author{
Addass, P.A ${ }^{1}$ \\ Adamawa State University, Animal Production Department, P.M.B 25 Mubi Adamawa \\ State.Nigeria \\ Corresponding Author: paaddass@yahoo.com
}

\begin{abstract}
The common Nigerian bull cattle breeds, Rahaji (RJ), Bunaji (BJ), Sokoto Gudali (SG) and Adamawa Gudali (AG) were randomly selected as they were coming into Mubi main abattoir from different background for slaughter. Previous sexual activities were not monitored. The study was carried out to assess the effect of age and body condition scores (BCS) on gonodal and extragonodal sperm reserve. Significant age group differences on sperm production were observed. Age group $\geq 4$ years had highest sperm production potential followed by age group $3 \frac{1}{2}$-4years, $1 \frac{1}{2}-2$ years while $2 \frac{1}{2}$-3years age group had the least value. Respective paired testes sperm reserve (PTSR) values were; $136.66 \pm 2.19 \times 10^{9}, 130.52 \pm 2.21 \times 10^{9}, 123.35 \pm 4.47 \times 10^{9}$ and $120.95 \pm 2.66 \times 10^{9}$; right testes sperm reserve (RTSR) corresponding values were; $68.36 \pm 1.10 \times 10^{9}, 65.60 \pm 1.11 \times 10^{9}, 60.69 \pm 2.25 \times 10^{9}$ and $59.25 \pm 1.34 \times 10^{9}$; Values for left testes sperm reserve (LTSR) $66.37 \pm 1.42 \times 10^{9}, 64.15 \pm 1.44 \times 10^{9}, 62.92 \pm 2.91 \times 10^{9}$ and $61.76 \pm 1.73 \times 10^{9}$, while values for paired epididymal sperm reserve (PESR) were; $276.65 \pm 3.81 \times 10^{9}$, $264.87 \pm 3.84 \times 10^{9}, 253.40 \pm 7.27 \times 10^{9}$, and $241.89 \pm 4.63 \times 10^{9}$. significant $(P<0.001)$ BCS difference on sperm count was depicted indicating $\mathrm{BCS}_{5}$ having the highest values followed by BCS4, and 3 while BCS2 had the least. Corresponding values for PTSR were; $150.29 \pm 2.35 \times 10^{9}$, $133.64 \pm 2.00 \times 10^{9}, 122.23 \pm 2.41 \times 10^{9}$ and $98.55 \pm 3.98 \times 10^{9}$; RTSR values were; $75.78 \pm 1.44 \times 10^{9}$, $66.19 \pm 1.01 \times 10^{9}, 61.31 \pm 1.22 \times 10^{9}$ and $47.51 \pm 2.01 \times 10^{9}$; for LTSR values were; $73.04 \pm 1.85 \times 10^{9}$, $66.21 \pm 1.30 \times 10^{9}, 60.85 \pm 1.57 \times 10^{9}$ and $50.84 \pm 2.59 \times 10^{9}$ while PESR corresponding values were; $303.59 \pm 4.96 \times 10^{9}, 267.92 \pm 3.49 \times 10^{9}, 245.72 \pm 4.20 \times 10^{9}$ and $211.16 \pm 6.92 \times 10^{9}$. Generally it was observed that higher age group as well as BCS5 had a better potential for sperm production among the bull breeds studied.
\end{abstract}

Keywords: Bull, Sperm, Age group, Body condition score.

\section{DESCRIPTION OF THE PROBLEM}

It is known that breed $s$ of Nigerian cattle grow slowly, taking up to 4 years to reach sexual maturity and attain slaughter weight with low dressing percentage (Oyenuga, 1982). In an attempt to improve the genetic potentials of the indigenous cattle breeds, exotic breeds were imported to cross breed with the local breeds, however wiped out by streptothricosis. Exploitation of indigenous breeds becomes inevitable. It is on these bases that the study was conducted to evaluate the sperm production potentials of some selected indigenous breeds of cattle known to be tolerant to the dreaded diseases and environmental conditions, so as to be able to identify the suitable age and body condition score to exploit on the indigenous cattle. There is also an intention to address how producers can evaluate and manage bull fertility. Sperm production potentials of bulls are of paramount importance in the determination of male/female ratio during natural mating as well as in artificial insemination programme (Wildeus and Entwistle, 1982). It is generally recognized that poor sperm production potential is one of the major factors limiting beef production in the tropics. A nation like Nigeria that continuously waste its livestock genetic resources will continue to grope and be at the mercy of other nations, who use it as dumping ground for their prowess in the ability to create employment, alleviate poverty, eliminate protein mal-nutrition and provide for social needs of their human heritage. In essence Nigeria's cattle breeds are a neglected heritage that need to be exploited more. Nigeria is endowed with about 13.8 million cattle, $97 \%$ of which are traditional Zebu breeds besides other livestock species, yet average 
Nigerians consumes less than $25 \%$ of the recommended $34 \mathrm{~g} /$ head/day of animal protein.

\section{MATERIALS AND METHODS}

The common Nigerian cattle breeds; Rahaji, Bunaji, Sokoto Gudali, and Adamawa Gudali were used for the study. They were randomly selected as they were coming into the slaughtering slab of mubi main abattoir . dentition methods as described by Philips (1977), Sastry and Thomas (1980) and McNitt(1983) were adopted for age determination in the bulls. Body condition score (scale 0-5) as described by Jefferies (1961), Lowman et al, (1976) and Pullan, (1978) taken before slaughter was used in the study. Hemocytometer, phase contrast microscope and cell counter were used for sperm count after prepared samples of testes and epididymedes were introduced into the neubeur hemocytometer counting chamber. Sperm cells of each sample were counted twice for certainty and accuracy.

\section{RESULT AND DDISCUSSION}

Significant age group variability on sperm production was evident among the various age groups. Age group $\geq 4$ years had highest potentials as; PTSR $\left(136.66 \pm 2.19 \times 10^{9}\right), \operatorname{RTSR}\left(68.36 \pm 1.10 \times 10^{9}\right)$, LTSR $\left(66.37 \pm 1.42 \times 10^{9}\right)$, PESR $\left(276.65 \pm 3.81 \times 10^{9}\right)$, PCSR $\left(118.65 \pm 1.81 \times 10^{9}\right), \quad$ PCoSR $\left(21.32 \pm 1.04 \times 10^{9}\right)$ and PCaSR $\left(135.04 \pm 2.03 \times 10^{9}\right)$. Age groups $1 \frac{1}{2}$-2years and $3 \frac{1}{2}-4$ years had similar sperm production potentials, while age group $2 \frac{1}{2}-3$ years had the least as thus: PTSR $\left(120.95 \pm 2.66 \times 10^{9}\right)$, RTSR $\left(59.25 \pm 1.34 \times 10^{9}\right), \quad$ LTSR $\left(61.76 \pm 1.73 \times 10^{9}\right)$, PESR $\left(241.89 \pm 4.63 \times 10^{9}\right), \quad$ PCSR $\quad\left(107.32 \pm 2.20 \times 10^{9}\right)$, PCaSR $\left(116.69 \pm 2.47 \times 10^{9}\right)$.

Significant $(P<0.001)$ age group differences on SC was evident indicating age group $\geq 4$ years with the largest $(31.99 \pm 0.12 \mathrm{~cm})$ scrotal circumference, followed by age group $31 / 2-4$ years $(30.37 \pm 0.12 \mathrm{~cm})$ then $1 \frac{1}{2}-2$ years age group $(29.35 \pm 0.25 \mathrm{~cm})$ while age group of $2 \frac{1}{2}-3$ years had the smallest $(28.26 \pm 0.15 \mathrm{~cm})$. Significant age group variation on FPEC was also observed with bulls of age group $2 \frac{1}{2}$ - 3 years were found most vulnerable $(16.50 \pm 0.70)$ followed by bulls of age group $3 \frac{1}{2^{-}} 4$ years $(14.62 \pm 0.58)$ while age groups $1 \frac{1}{2}-2$ years and $\geq$ 4years were least infected $(9.56 \pm 1.18$ and $10.82 \pm 0.58$ ) respectively. (Table 1$)$.

\begin{tabular}{|c|c|c|c|c|c|c|c|c|c|c|}
\hline Variable & $\mathbf{N}$ & $\begin{array}{l}\text { PTSR } \\
\left(\times 10^{9}\right)\end{array}$ & $\begin{array}{l}\text { RTSR } \\
\left(\times 10^{9}\right)\end{array}$ & $\begin{array}{l}\text { LTSR } \\
\left(\times 10^{9}\right)\end{array}$ & $\begin{array}{l}\text { PESR } \\
\left(\times 10^{9}\right)\end{array}$ & $\begin{array}{l}\text { PCSR } \\
\left(\times 10^{9}\right)\end{array}$ & $\begin{array}{c}\text { PCoSR } \\
\left(\times 10^{9}\right)\end{array}$ & $\begin{array}{c}\text { PCaSR } \\
\left(\times 10^{9}\right)\end{array}$ & $\mathrm{SC}(\mathrm{cm})$ & FPEC \\
\hline Age & & $\star * * *$ & $\star \star \star \star *$ & * & $\star * * *$ & ** & * & $\star * * *$ & $* * \star$ & $\star * * *$ \\
\hline $\begin{array}{l}1 \frac{1}{2} 2^{-} \\
2 \text { years }\end{array}$ & $\begin{array}{l}1 \\
5\end{array}$ & $\begin{array}{l}123.35 \pm \\
4.47^{\text {bc }}\end{array}$ & $\begin{array}{l}60.69 \pm \\
2.25^{\mathrm{bc}}\end{array}$ & $\begin{array}{l}62.92 \pm \\
2.92^{\mathrm{ab}}\end{array}$ & $\begin{array}{l}253.40 \pm \\
7.27^{\mathrm{bc}}\end{array}$ & $\begin{array}{l}109.38 \pm \\
3.69^{\text {bc }}\end{array}$ & $\begin{array}{l}17.00 \pm \\
2.12^{\mathrm{c}}\end{array}$ & $\begin{array}{l}126.22 \pm \\
4.15^{\mathrm{b}}\end{array}$ & $\begin{array}{l}29.35 \pm \\
0.25^{c}\end{array}$ & $\begin{array}{l}9.56 \pm \\
1.18^{\mathrm{c}}\end{array}$ \\
\hline $\begin{array}{l}21 / 2^{-} \\
\text {3years }\end{array}$ & $\begin{array}{l}4 \\
3 \\
\end{array}$ & $\begin{array}{l}120.95 \pm \\
2.66^{c}\end{array}$ & $\begin{array}{l}59.25 \pm \\
1.34 c\end{array}$ & $\begin{array}{l}61.76 \pm \\
1.73^{b}\end{array}$ & $\begin{array}{l}241.89 \pm \\
4.63^{c}\end{array}$ & $\begin{array}{l}107.32 \pm \\
2.20^{\mathrm{C}}\end{array}$ & $\begin{array}{l}17.92 \pm \\
1.26^{\mathrm{c}}\end{array}$ & $\begin{array}{l}116.69 \pm \\
2.47^{c}\end{array}$ & $\begin{array}{l}28.26 \pm \\
0.15^{d}\end{array}$ & $\begin{array}{l}16.50 \pm \\
0.70^{\mathrm{a}} \\
\end{array}$ \\
\hline $\begin{array}{l}3 \% / 2- \\
4 y e a r s\end{array}$ & $\begin{array}{l}6 \\
4 \\
\end{array}$ & $\begin{array}{l}130.52 \pm \\
2.21^{\mathrm{b}}\end{array}$ & $\begin{array}{l}65.60 \pm \\
1.11^{b}\end{array}$ & $\begin{array}{l}64.15 \pm \\
1.44^{\mathrm{ab}}\end{array}$ & $\begin{array}{l}264.87 \pm \\
3.84^{\text {b }}\end{array}$ & $\begin{array}{l}116.54 \pm \\
1.83^{a}\end{array}$ & $\begin{array}{l}18.75 \pm \\
1.05^{b}\end{array}$ & $\begin{array}{l}129.77 \pm \\
2.05^{b}\end{array}$ & $\begin{array}{l}30.37 \pm \\
0.12^{b}\end{array}$ & $\begin{array}{l}14.62 \pm \\
0.58^{b}\end{array}$ \\
\hline $\begin{array}{l}\geq 4 \\
\text { years }\end{array}$ & $\begin{array}{l}6 \\
7 \\
\end{array}$ & $\begin{array}{l}136.66 \pm \\
2.19^{\mathrm{a}}\end{array}$ & $\begin{array}{l}68.36 \pm \\
1.10^{\mathrm{a}}\end{array}$ & $\begin{array}{l}66.37 \pm \\
1.42 a\end{array}$ & $\begin{array}{l}276.65 \pm \\
3.81^{\mathrm{a}}\end{array}$ & $\begin{array}{l}118.65 \pm \\
1.81^{\mathrm{a}}\end{array}$ & $\begin{array}{l}21.32 \pm \\
1.04^{a}\end{array}$ & $\begin{array}{l}135.04 \pm \\
2.03^{\mathrm{a}}\end{array}$ & $\begin{array}{l}31.99 \pm \\
0.12^{\mathrm{a}}\end{array}$ & $\begin{array}{l}10.82 \pm \\
0.50^{c}\end{array}$ \\
\hline
\end{tabular}

Table1: Means \pm SE by age of testes and Paired epididymal sperm reserve count of bull cattle breeds

$\mathrm{N}=$ Number of observation, ${ }^{*}=\mathrm{P}<0.05,{ }^{* *}=\mathrm{P}<0.01,{ }^{* * *}=\mathrm{P}<0.001$. PTSR=Paired Testes Sperm Reserve, RTSR=Right Testes Sperm Reserve, LTSR=Left Testes Sperm Reserve, PESR=Paired epididymal Sperm reserve, PCSR=Paired Carput Sperm Seserve, PCoSR=Paired Corpus Sperm Reserve, PCaSR=Paired Cauda Sperm Reserve, SC=scrotal circumference, FPEC=Feacal Parasitic Egg Count. Note: Means for groups in homogenous subsets and with same super script(s) are statically similar.

Significant BCS variations among the breeds of bulls were made pronounced on sperm production potentials indicating BCS5 with the highest values; PTSR $\left(150.29 \pm 2.35 \times 10^{9}\right)$, RTSR $\left(75.78 \pm 1.44 \times 10^{9}\right)$ LTSR $\left(73.04 \pm 1.85 \times 10^{9}\right)$, PESR $\left(303.59 \pm 4.96 \times 10^{9}\right)$, PCSR $\left(133.20 \pm 2.35 \times 10^{9}\right)$ and PCaSR $\left(146.71 \pm 2.65 \times 10^{9}\right)$ followed by BCS4 then BCS3 while BSC2 had the lowest potentials for sperm production as follows: PTSR $\left(98.55 \pm 3.98 \times 10^{9}\right)$, RTSR $\left(47.51 \pm 2.01 \times 10^{9}\right), \quad$ LTSR $\left(50.84 \pm 2.59 \times 10^{9}\right), \quad$ PESR $\left(211.16 \pm 6.92 \times 10^{9}\right), \quad \operatorname{PCSR} \quad\left(89.89 \pm 3.29 \times 10^{9}\right)$ and PCaSR $\left(102.03 \pm 3.69 \times 10^{9}\right)$. Significant $(P<0.001)$ BCS variation among the bulls on SC was recorded. Bulls of BCS5 had the largest $(31.44 \pm 0.16 \mathrm{~cm}) \mathrm{SC}$, followed by BCS4, while those of the BCS2 had the least $(28.66 \pm 0.22 \mathrm{~cm}) \quad S C$. Significant $(P<0.001)$ FPEC was noticed on BCS indicating highest infection in bulls with BCS2 $(21.83 \pm 1.05)$ and lowest on BCS5 (9.39 \pm 0.75$)$. (Table 2). 
Agric. Biol. J. N. Am., 2011, 2(2): 203-206

Table 2. Means \pm SE by BCS of Testes and paired epididymal sperm Reserve count of bull cattle.

\begin{tabular}{|c|c|c|c|c|c|c|c|c|c|c|}
\hline Variable & $\mathbf{N}$ & $\begin{array}{r}\text { PTSR } \\
\left(\times 10^{9}\right) \\
\end{array}$ & $\begin{array}{l}\text { RTSR } \\
\left(\times 10^{9}\right) \\
\end{array}$ & $\begin{array}{r}\text { LTSR } \\
\left(\times 10^{9}\right) \\
\end{array}$ & $\begin{array}{l}\text { PESR } \\
\left(\times 10^{9}\right) \\
\end{array}$ & $\begin{array}{r}\text { PCSR } \\
\left(\times 10^{9}\right) \\
\end{array}$ & $\begin{array}{c}\text { PCoSR } \\
\left(\times 10^{9}\right) \\
\end{array}$ & $\begin{array}{c}\text { PCaSR } \\
\left(\times 10^{9}\right) \\
\end{array}$ & $\mathrm{SC}(\mathrm{cm})$ & FPEC \\
\hline BCS & & $\star \star \star *$ & $\star * *$ & $* * *$ & $* * *$ & $\star * *$ & NS & $* * *$ & $* * *$ & $* * *$ \\
\hline 2 & 19 & $\begin{array}{l}98.55 \pm 3 . \\
98^{d}\end{array}$ & $\begin{array}{l}47.51 \pm \\
2.01^{\mathrm{d}}\end{array}$ & $\begin{array}{l}50.84 \pm \\
2.59^{d}\end{array}$ & $\begin{array}{l}211.16 \pm 6 \\
.92^{\mathrm{d}}\end{array}$ & $\begin{array}{l}89.89 \pm \\
3.29^{d}\end{array}$ & $\begin{array}{l}19.36 \pm \\
1.88^{a}\end{array}$ & $\begin{array}{l}102.03 \pm \\
3.69^{d}\end{array}$ & $\begin{array}{l}28.66 \pm \\
0.22^{d}\end{array}$ & $\begin{array}{l}21.83 \pm \\
1.05^{\mathrm{a}}\end{array}$ \\
\hline 3 & 53 & $\begin{array}{l}122.23 \pm 2 \\
.41^{\mathrm{C}}\end{array}$ & $\begin{array}{l}61.31 \pm 1 . \\
22^{c}\end{array}$ & $\begin{array}{l}60.85 \pm \\
1.57^{c}\end{array}$ & $\begin{array}{l}245.72 \pm 4 \\
.20^{C}\end{array}$ & $\begin{array}{l}106.04 \pm 2 \\
.00^{\mathrm{C}}\end{array}$ & $\begin{array}{l}16.54 \pm 1 . \\
14^{\mathrm{a}}\end{array}$ & $\begin{array}{l}121.10 \pm \\
2.24^{\mathrm{c}}\end{array}$ & $\begin{array}{l}29.86 \pm \\
0.14^{\mathrm{C}}\end{array}$ & $\begin{array}{l}14.95 \pm \\
0.64^{b}\end{array}$ \\
\hline 4 & 78 & $\begin{array}{l}133.64 \pm 2 \\
.00^{\mathrm{b}}\end{array}$ & $\begin{array}{l}66.19 \pm 1 . \\
01^{\mathrm{b}}\end{array}$ & $\begin{array}{l}66.21 \pm \\
1.30^{\mathrm{b}}\end{array}$ & $\begin{array}{l}267.92 \pm 3 \\
.49^{\mathrm{b}}\end{array}$ & $\begin{array}{l}117.96 \pm 1 \\
.66^{\mathrm{b}}\end{array}$ & $\begin{array}{l}19.03 \pm 0 . \\
95^{\mathrm{a}}\end{array}$ & $\begin{array}{l}131.09 \pm \\
1.86^{\mathrm{b}}\end{array}$ & $\begin{array}{l}30.45 \pm \\
0.11^{\mathrm{b}}\end{array}$ & $\begin{array}{l}11.60 \pm \\
0.53^{\mathrm{c}}\end{array}$ \\
\hline 5 & 39 & $\begin{array}{l}150.29 \pm 2 \\
.35^{\mathrm{a}}\end{array}$ & $\begin{array}{l}75.78 \pm 1 . \\
44^{\mathrm{a}}\end{array}$ & $\begin{array}{l}73.04 \pm \\
1.85^{\mathrm{a}}\end{array}$ & $\begin{array}{l}303.59 \pm 4 \\
.96^{\mathrm{a}}\end{array}$ & $\begin{array}{l}133.20 \pm 2 \\
.35^{\mathrm{a}}\end{array}$ & $\begin{array}{l}23.30 \pm 1 . \\
35^{\mathrm{a}}\end{array}$ & $\begin{array}{l}146.71 \pm \\
2.65^{\mathrm{a}}\end{array}$ & $\begin{array}{l}31.44 \pm \\
0.16^{\mathrm{a}}\end{array}$ & $\begin{array}{l}9 . .39 \pm \\
0.75^{d}\end{array}$ \\
\hline
\end{tabular}

$\mathrm{N}=$ Number of observation, NS= Not significant, ${ }^{* * *}=\mathrm{P}<0.001, \mathrm{BCS}=$ Body Condition Score. PTSR=Paired Testes Sperm Reserve, RTSR=Right Testes Sperm Reserve, LTSR=Left Testes Sperm Reserve, PESR=Paired epididymal Sperm reserve, PCSR=Paired Carput Sperm Seserve, PCoSR=Paired Corpus Sperm Reserve, PCaSR=Paired Cauda Sperm Reserve, SC=scrotal circumference, FPEC=Feacal Parasitic Egg Count.

Note: Means for groups in homogenous subsets and with same super script(s) are statically similar.

The significant increase in sperm production with age discovered in this study agreed with the findings of Ashdown and Hancock, (1980) who reported an increase in sperm production with age up to seven years in bulls. In general SC, ejaculate volume, total number of spermatozoa and number of viable spermatozoa increases with age in bulls (Britto, et al., 2002). These findings are in agreement with, is study where age group $\geq 4$ years had the highest sperm production potential as well as the SC in bulls.

In support of the findings of this study where bulls of $\geq 4$ years were least infected with parasites than the younger bulls of 2- 4 years was the report of Andrew, (1980) and Oni et al (1988) who discovered that the nature of cattle feeding and experience for feed selection which exposes animals to parasitic infection increases with age.

Body condition score variability was made pronounced on sperm production potentials and SC indicating BCS5 with highest sperm reserve count as well as large SC. Opposite was the case on faecal parasitic count with the BCS2 recording highest infection. Body size and semen producing capacity are difficult to separate (Salisbury, et al., 1978). This statement support this findings where higher BCS had higher sperm count unlike feacal parasitic load which was indicative of lowest BCS;Scrotal circumference measurements has been used to predict sperm producing capacity in young bulls (Han, et al., 1976). There is a direct linear relationship between SC and testicular weight, length and width (Osinowo, et al., 1977, Akingbemi and Aire, 1990, Tegegne et al 1992a). Generally sperm production increases in bulls at an increasing age and body condition scores suggesting that breeding bulls (natural/artificial) should attain full maturity age and at a higher body condition scores, however not more than 7 years of age at the time testes sperm producing tissues will begin to degenerates (Tegegne, et al., 1992a).

\section{REFERENCES}

Akingbemi, B.A and Aire, T.A (1990). Some reproductive parameters of Sokoto Gudali (Bos indicus) bulls. Journal of Animal Production Research, 10 (2): 121133.

Andrew F.F (1980). Farm Animal behavior, The English language book Society and Bailiere Tindali New York. $2^{\text {nd }}$ edition pp 220.

Ashdown, R.R and Hancock, J.L (1980). Functional anatomy of male reproduction. In hafez, E.S.E Reproduction in farm animals. Ea and Febiger, Philadelphia, USA pp 7-29.

Brito, L.F., Silva, A.E., Deragon, L.A and Kastelic, J.P. (2002). Effect of age and genetic group on characteristics of the scrotum testes and testicular vascular cones and sperm production and semen quality in Al bulls. Thenogenology, (2002).

Hahn, J., Foote, R.K and Seidel, Jr., G.E (1976). Testicular growth and related sperm sperm out put in dairy bulls Journal of animal science, 29: 41-47.

Jefferies, B.C. (1961). Body condition scoring and its use in management. Tesmanian. Journal of agriculture, 32: 19-21.

Lowman, B.G., Scott, N.H. and Somorville, S.H (1976). Condition scoring of cattle. The East of Scotland collage of agriculture bulletin, No.6, Edinburg.

McNitt, J.I. (1983). Livestock Husbandry techniques, Low priced edition. Granada publishing company limited, Frogmore, St. Albans A122 NF and 36 Golden square, London WIR 4AH (1983). 
Agric. Biol. J. N. Am., 2011, 2(2): 203-206

Oni, O.O., Buvanendran, V. and Dim, N.I (1988). The influence of some environmental on the growth rate of two Nigerian cattle breeds and hybrid with the Charolais. Journal of animal production research. 8(2): 121-131.

Osinowo, O.A., Denis, S.M., Osori, D.I.K and Molakan, E.C.I (1977). Scrotal and testicular dimensions in some West African bulls. Nigerian Journal of animal Production 4 (2): 31-36.

Oyenuga, V.A (1982). Future of the beef Industry in Nigeria, Proceedings of the National conference on beef production, Kaduna, Nigeria, July, 27-30.

Philips, T.A. (1977). An Agricultural note book with special references to Nigeria, New edition Longman group Ltd. London.

Pullan, N.B. (1978). Condition score of white Fulani cattle. Tropical animal Health Production, 10: 118-120.
Salisbury, G.W., Vandemark, N.L and Lodge, J.R (1978). Physiology of reproduction and Artificial Insemination of cattle. Freeman, W.H. (ed). Sanfrancisco, pp574.

Sastry S.R and Thomas C.K. (1980). Dentition in farm animal management. In: farm animal management. VIKAS publishing House PVT, Ltd. India pp. 39-45.

Tegegne, A., Enstwistle, K.W and Mukasa-Mugarwa, E (1992a). Gonodal and extragonodal sperm reserves and testicular histometrics characteristics in zebu and cross breed bulls. Effect of dry season nutritional supplementation. Animal reproduction science, 29: (12): 25-33.

Wildeus, S and Entwistle, K.W (1982). Post pubertal changes in gonodal and extragonodal sperm reserves in Bos indicus strain bulls. Thoriogenology 17: 655667. 